\title{
OSCILLATION OF THE SOLUTIONS OF A CLASS OF IMPULSIVE DIFFERENTIAL EQUATIONS WITH A DEVIATING ARGUMENT
}

\author{
D.D. BAINOV \\ Higher Medical Institute, P.O. Box 45 \\ Sofia-1504, Bulgaria \\ MARGARITA B. DIMITROVA \\ Technical University \\ Sliven, Bulgaria \\ ANGEL B. DISHLIEV \\ University of Technology \\ Sofia, Bulgaria
}

(Received January, 1995; Revised May, 1997)

Sufficient conditions are found for oscillation of all solutions of a class of impulsive differential equations with deviating argument.

Key words: Oscillation, Impulsive Differential Equations, Deviating Argument.

AMS subject classifications: $34 \mathrm{~A} 37$.

\section{Introduction}

Impulsive differential equations with deviating argument are an adequate mathematical apparatus for simulation of processes which depend on their prehistory and are subject to short-time disturbances. Such processes occur in the theory of optimal control, theoretical physics, population dynamics, pharmacokinetics, biotechnologies, industrial robotics, economics, etc. In spite of numerous possibilities for their applications, the theory of these equations is developing rather slowly due to difficulties of theoretical and technical nature.

In the recent twenty years, a large number of studies devoted to the oscillation were published. To the best of our knowledge, no other publications on this subject have every been published.

In the present paper, we establish sufficient conditions for oscillation of all solutions of a class of impulsive differential equations with fixed moments of impulse effect and a deviating argument.

Let us note that in contrast to [1], the present paper deals with the oscillatory properties of more general non-homogeneous impulsive differential-difference 
equation.

\section{Preliminary Notes}

Let $h \in \mathbb{R}_{+}=(0, \infty) ; p, q \in C\left(\overline{\mathbb{R}}+, \mathbb{R}_{+}\right), \overline{\mathbb{R}}_{+}=[0, \infty),\left\{\tau_{k}\right\}_{k=1}^{\infty}$ be a monotone increasing unbounded sequence of nonnegative numbers, and $\left\{b_{k}\right\}_{k=1}^{\infty}$ be a sequence of real numbers.

Consider the following impulsive differential equations with a deviating argument

$$
\left.\begin{array}{c}
x^{\prime}(t)+q(t) x(t)+p(t) x(t-h)=0, \quad t \neq \tau_{k} \\
\Delta x\left(\tau_{k}\right)=x\left(\tau_{k}+0\right)-x\left(\tau_{k}\right)=b_{k} x\left(\tau_{k}\right)
\end{array}\right\}
$$

with the initial condition

$$
x(t)=\varphi(t), \quad-h \leq t \leq 0,
$$

where $\varphi \in C([-h, 0], \mathbb{R})$.

We construct the sequence

$$
\left\{t_{i}\right\}_{i=1}^{\infty}=\left\{\tau_{i}\right\}_{i=1}^{\infty} \cup\left\{\tau_{i h}\right\}_{i=1}^{\infty}
$$

where $\tau_{i h}=\tau_{i}+h$ and $t_{i}<t_{i+1}$ for $i \in \mathbb{N}$.

Definition 1: By a solution of the equation (1) with initial function (3), we mean any function $x:[-h, \infty) \rightarrow \mathbb{R}$, for which the following conditions are valid:

1. If $-h \leq t \leq 0$, then $x(t)=\varphi(t)$.

2. If $0 \leq t \leq t_{1}=\tau_{1}$, then $x$ coincides with the solution of the equation

$$
x^{\prime}(t)+q(t) x(t)+p(t) x(t-h)=0 .
$$

3. If $t_{i}<t \leq t_{i+1}, \quad t_{i} \in\left\{\tau_{i}\right\}_{i=1}^{\infty} \backslash\left\{\tau_{i h}\right\}_{i=1}^{\infty}$, then $x$ coincides with the solution of the problem

$$
\begin{gathered}
x^{\prime}(t)+q(t) x(t)+p(t) x(t-h)=0 \\
x\left(t_{i}+0\right)=\left(1+b_{k_{i}}\right) x\left(t_{i}\right),
\end{gathered}
$$

where the number $k_{i}$ is determined from the equality $t_{i}=\tau_{k}$.

4. If $t_{i}<t \leq t_{i+1}, \quad t_{i} \in\left\{\tau_{i h}\right\}_{i=1}^{\infty} \backslash\left\{\tau_{i}\right\}_{i=1}^{\infty}$, then $x$ coincides with the solution of the problem

$$
\begin{gathered}
x^{\prime}(t)+q(t) x(t+0)+p(t) x(t-h+0)=0 \\
x\left(t_{i}+0\right)=x\left(t_{i}\right) .
\end{gathered}
$$

5. If $t_{i}<t \leq t_{i+1}, \quad t_{i} \in\left\{\tau_{i}\right\}_{i=1}^{\infty} \cap\left\{\tau_{i h}\right\}_{i=1}^{\infty}$, then $x$ coincides with the solution of the problem 


$$
\begin{gathered}
x^{\prime}(t)+q(t) x(t+0)+p(t) x(t-h+0)=0 \\
x\left(t_{i}+0\right)=\left(1+b_{k_{i}}\right) x\left(t_{i}\right)
\end{gathered}
$$

where $k_{i}$ is determined from the equality $t_{i}=\tau_{k_{i}}$.

The definition of a solution of the problem (2), (3) is analogous to Definition 1, where $b:[0, \infty) \rightarrow \mathbb{R}$.

Definition 2: A nonzero solution $x$ of the equation (1) is said to be nonoscillating if there exists a point $t_{0} \geq 0$ such that $x(t)$ has a constant sign for $t \geq t_{0}$. Otherwise, the solution $x$ is said to oscillate.

In work [1], the oscillatory properties of the impulsive differential equation with a deviating argument,

$$
\left.\begin{array}{c}
x^{\prime}(t)+p(t) x(t-h)=0, \quad t \neq \tau_{k} \\
\Delta x\left(\tau_{k}\right)=b_{k} x\left(\tau_{k}\right),
\end{array}\right\}
$$

are studied. To make this presentation self-contained, we formulate the most significant results of [1].

Theorem 1: [1] Let the following conditions hold:

1. $p \in C\left(\overline{\mathbb{R}}_{+}, \mathbb{R}_{+}\right)$.

2. There exists a constant $T>0$ such that for any $k \in \mathbb{N}$ we have $\tau_{k+1}$ -

3. $\quad \limsup _{k \rightarrow \infty} \frac{1}{1+b_{k}} \int_{\tau_{k}}^{\tau_{k}+\Delta} p(s) d s>1$, where $\Delta=\min (h, T)$.

Then all solutions of the equation (4) oscillate.

Theorem 2: [1] Let the following conditions hold:

1. $p \in C\left(\overline{\mathbb{R}}_{+}, \mathbb{R}_{+}\right)$.

2. There exists a constant $T>h>0$ such that for any $k \in \mathbb{N}$ we have $\tau_{k+1}-\tau_{k} \geq T$.

3. There exists a constant $M>0$ such that for any $k \in \mathbb{N}$ we have $0 \leq b_{k} \leq$

4. $\quad \liminf _{t \rightarrow \infty} \int_{t-h}^{t} p(s) d s>\frac{1+M}{e}$.

Then all solutions of the equation (4) oscillate.

Introduce the following conditions:

H1. There exists a positive constant $T$ such that for any $k \in \mathbb{N}$ we have $\tau_{k+1}-\tau_{k} \geq T>h$.

H2. $\quad b_{k} \neq-1$ for all $k \in \mathbb{N}$.

H3. $b_{k}>-1$ for all $k \in \mathbb{N}$.

\section{Main Results}

Theorem 3: Let the following conditions hold:

1. Conditions II1 and $\mathrm{H} 2$ are satisfied.

2. $q(t)+\frac{1}{1+b_{k}} p(t)>0, t \in \overline{\mathbb{R}}_{+}, k \in \mathbb{N}$.

Then all solutions of the problem (1), (3) (i.e., the problem described by equation 
(1) with the initial condition (3)) oscillate.

Proof: Let a nonoscillating solution $x$ of problem (1), (3) exist. Without loss of generality, we may assume that $x(t)>0$ for $t \geq t_{0} \geq 0$. Then $x(t-h)>0$ for $t \geq t_{0}+h$ too.

From (1) it follows that $x(t)$ is a decreasing function on the set $\left(t_{0}+h, \tau_{s}\right) \cup$ $\left[\bigcup_{i=s}^{\infty}\left(\tau_{i}, \tau_{i+1}\right)\right], \tau_{s-1}<t_{0}+h<\tau_{s}$.

We integrate (1) from $\tau_{k}$ to $\tau_{k}+h(k \geq s)$ and obtain that

$$
\begin{gathered}
x\left(\tau_{k}+h\right)-x\left(\tau_{k}+0\right)+\int_{\tau_{k}}^{\tau_{k}+h} q(s) x(s) d s+\int_{\tau_{k}}^{\tau_{k}+h} p(s) x(s-h) d s=0, \\
x\left(\tau_{k}+h\right)-x\left(\tau_{k}+0\right)+\inf _{s \in\left[\tau_{k}, \tau_{k}+h\right]} x(s) \int_{\tau_{k}}^{\tau_{k}+h} q(s) d s \\
+\inf _{s \in\left[\tau_{k}, \tau_{k}+h\right]} x(s-h) \int_{\tau_{k}} p(s) d s \leq 0,
\end{gathered}
$$

and

$$
x\left(\tau_{k}+h\right)-x\left(\tau_{k}+0\right)+x\left(\tau_{k}+h\right) \int_{\tau_{k}}^{\tau_{k}+h} q(s) d s+x\left(\tau_{k}\right) \int_{\tau_{k}}^{\tau_{k}+h} p(s) d s \leq 0 .
$$

Since $x\left(\tau_{k}\right)=\frac{x\left(\tau_{k}+0\right)}{1+b_{k}}$ and $x\left(\tau_{k}+h\right)<x\left(\tau_{k}+0\right)$, then from (5) it follows that

$$
\left.\begin{array}{c}
x\left(\tau_{k}+h\right)\left[1+\int_{\tau_{k}}^{\tau_{k}+h} q(s) d s\right]+x\left(\tau_{k}+0\right)\left[\frac{1}{1+b_{k}} \int_{\tau_{k}}^{\tau_{k}+h} p(s) d s-1\right] \leq 0 \\
x\left(\tau_{k}+h\right) \int_{\tau_{k}}^{\tau_{k}+h}\left[q(s)+\frac{1}{1+b_{k}} p(s)\right] d s \leq 0 .
\end{array}\right\}
$$

The last inequality contradicts condition 2 of Theorem 3 .

Corollary 1: Let the following conditions hold:

1. Conditions $\mathrm{H} 1$ and $\mathrm{H} 2$ are satisfied.

2. $\quad \limsup _{k \rightarrow \infty} \frac{1}{1+b_{k}} \int_{\tau_{k}}^{\tau_{k}+h} p(s) d s>1$.

Then all solutions of the problem (1), (3) oscillate.

Proof: Analogously to the proof of Theorem 3, we obtain (6) which implies

$$
x\left(\tau_{k}+0\right)\left[\frac{1}{1+b_{k}} \int_{\tau_{k}}^{\tau_{k}+h} p(s) d s-1\right] \leq 0 .
$$


The last inequality contradicts condition 2 of Corollary 1.

Corollary 2: Let conditions $\mathrm{H} 1$ and $\mathrm{H} 3$ hold.

Then all solutions of the problem (1),(3) oscillate.

Proof: From $\mathrm{H} 3$ it follows that $\frac{1}{1+b_{k}}>0$. From the condition that $p, q \in$ $C\left(\overline{\mathbb{R}}_{+}, \mathbb{R}_{+}\right)$we conclude that

$$
q(t)+\frac{1}{1+b_{k}} p(t)>0, t \in \overline{\mathbb{R}}_{+}, k \in \mathbb{N} .
$$

Then from Theorem 3, it follows that all solutions of the problem (1), (3) oscillate.

We shall carry out equivalent transformations of impulsive equation (1). Set

$$
x(t)=e^{-\int_{t_{0}}^{t} q(s) d s} z(t), \quad t \geq t_{0} \geq 0
$$

and substitute it into (1) to obtain

$$
z^{\prime}(t)+p_{1}(t) z(t-h)=0, \quad t \neq \tau_{k}
$$

where

$$
p_{1}(t)=p(t) \exp \left[\int_{t-h}^{t} q(s) d s\right]
$$

From the second equation in (1) it follows that

$$
\Delta z\left(\tau_{k}\right)=b_{k} z\left(\tau_{k}\right)
$$

Hence the equation (1) is equivalent to (7), (8). Then, Theorem 1 and Theorem 2 can be applied to impulsive differential equation (7), (8), and to (1), respectively.

Theorem 4: Let the following conditions hold:

1. There exists a positive constant $T$ such that for $k \in \mathbb{N}$ we have $\tau_{k+1}$

2. $\quad \begin{aligned} \tau_{k} \geq T . \\ b_{k} \neq-1, k \in \mathbb{N} .\end{aligned}$

3. $\quad \limsup _{k \rightarrow \infty} \frac{1}{1+b_{k}} \int{ }_{\tau_{k}}^{\tau_{k}+\Delta} p(s) \exp \left[\int_{s-h}^{s} q\left(s_{1}\right) d s_{1}\right] d s>1$, where $\Delta=\min (h, T)$.

Then all solutions of the problem (1), (3) oscillate.

Theorem 5: Let the following conditions hold:

1. Condition $\mathrm{H} 1$ is valid.

2. There exists a constant $M>0$ such that for any $k \in \mathbb{N}$, the inequalities $0 \leq b_{k} \leq M$ are valid.

3. $\quad \liminf _{t \rightarrow \infty} \int_{t-h}^{t} p(s) \exp \left[\int_{s-h}^{s} q\left(s_{1}\right) d s_{1}\right] d s>\frac{1+M}{e}$.

Then all solutions of the problem (1), (3) oscillate.

Consider the nonhomogeneous impulsive differential equation 


$$
\left.\begin{array}{c}
x^{\prime}(t)+p(t) x(t-h)=b(t), \quad t \neq \tau_{k} \\
\Delta x\left(\tau_{k}\right)=b_{k} x\left(\tau_{k}\right) .
\end{array}\right\}
$$

Lemma 1: Let the following conditions hold:

1. Condition $\mathrm{H} 1$ and condition 2 of Theorem 5 are valid.

2. There exists a function $w \in C^{1}\left(\overline{\mathbb{R}}_{+}, \overline{\mathbb{R}}_{+}\right)$such that $w^{\prime}(t)=b(t)$.

3. There exist two sequences $\left\{t_{k}^{\prime}\right\}_{k=1}^{\infty},\left\{t_{k}^{\prime \prime}\right\}_{k=1}^{\infty} \subset \mathbb{R}_{+}$and two constants $q_{1}, q_{2} \in \mathbb{R}+$ such that

(i) $\quad \lim _{k \rightarrow \infty} t_{k}^{\prime}=\lim _{k \rightarrow \infty} t_{k}^{\prime \prime}=\infty$,

(ii) $\quad w\left(t_{k}^{\prime}\right)=q_{1} \leq w(t) \leq q_{2}=w\left(t_{k}^{\prime \prime}\right), k \in \mathbb{N}, t \in \mathbb{R}_{+}$.

4. $\quad \liminf _{k \rightarrow \infty} \iint_{\tau_{k}}^{\tau_{k}+h} p(s) d s>1+M+\frac{M\left(q_{2}-q_{1}\right)}{S+q_{1}-q_{2}}, \quad$ where $\quad S=$ const, $\quad S>q_{2}-$ $q_{1}>0$.

Then all solutions of the problem (9), (3) satisfying the inequalities $\left|x\left(\tau_{k}\right)\right|>S$, $k \in \mathbb{N}$, oscillate.

Proof: Let $x(t)$ be a nonoscillating solution of the problem (9), (3). Suppose that $x(t)>0$ for $t \geq t_{0} \geq 0$. It is clear that $x(t-h)>0$ for $t \geq t_{0}+h$. Set

$$
z(t)=x(t)-w(t)+q_{1}
$$

and substitute it in (9). We obtain

$$
\left.\begin{array}{c}
z^{\prime}(t)+p(t) z(t-h) \leq 0, \quad t \neq \tau_{k} \\
\Delta z\left(\tau_{k}\right)=b_{k} z\left(\tau_{k}\right)+b_{k}\left(w\left(\tau_{k}\right)-q_{1}\right) .
\end{array}\right\}
$$

From condition 3 of Lemma 1, it follows that

$$
z\left(\tau_{k}\right)=x\left(\tau_{k}\right)-w\left(\tau_{k}\right)+q_{1} \geq S+q_{1}-q_{2}, \quad t \in \overline{\mathbb{R}}_{+} .
$$

We consider the following two cases:

Case 1: Let $z(t)>0$ be a solution of (11) for $t \geq t_{1} \geq t_{0}$. It is clear that $z(t-h)>0$ for $t \geq t_{1}+h$. From $p \in C\left(\overline{\mathbb{R}}_{+}, \mathbb{R}_{+}\right)$and $(1 \overline{1})$ it follows that $z$ is a decreasing function on the set $\left(t_{1}+h, \tau_{s}\right) \cup\left[\bigcup_{i=s}^{\infty}\left(\tau_{i}, \tau_{i+1}\right)\right]$ where the number $s$ is chosen so that $\tau_{s-1} \leq t_{1}+h<\tau_{s}$.

We integrate (11) to obtain

$$
z\left(\tau_{k}+h\right)-z\left(\tau_{k}+0\right)+\int_{\tau_{k}}^{\tau_{k}+h} p(s) z(s-h) d s \leq 0,
$$

whence, taking into account that $z\left(\tau_{k}+h\right)>0$ we obtain the inequality

$$
\inf _{s \in\left[\tau_{k}, \tau_{k}+h\right]} z(s-h) \int_{\tau_{k}}^{\tau_{k}+h} p(s) d s<z\left(\tau_{k}+0\right) .
$$

From (11) it follows that 


$$
z\left(\tau_{k}+0\right)=\left(1+b_{k}\right) z\left(\tau_{k}\right)+b_{k}\left(w\left(\tau_{k}\right)-q_{1}\right) .
$$

From (12) and (13) we obtain that

and

$$
z\left(\tau_{k}\right) \int_{\tau_{k}}^{\tau_{k}+h} p(s) d s \leq\left(1+b_{k}\right) z\left(\tau_{k}\right)+b_{k}\left[w\left(\tau_{k}\right)-q_{1}\right]
$$

$$
\int_{\tau_{k}}^{\tau_{k}+h} p(s) d s \leq 1+b_{k}+\frac{b_{k}\left[w\left(\tau_{k}\right)-q_{1}\right]}{z\left(\tau_{k}\right)} \leq 1+M+\frac{M\left(q_{2}-q_{1}\right)}{S+q_{1}-q_{2}} \text {. }
$$

The last inequality contradicts condition 4 of Lemma 1.

Case 2: Let $z(t)<0$ be a solution of (9) for $t \geq t_{1} \geq t_{0}$. From the substitution $(10)$, it follows that

$$
z\left(t_{k}^{\prime}\right)=x\left(t_{k}^{\prime}\right)-w\left(t_{k}^{\prime}\right)+q_{1}=x\left(t_{k}^{\prime}\right)
$$

But $x(t)>0$ for $t \geq t_{0}$, hence $x\left(t_{k}^{\prime}\right)>0$ for $t_{k}^{\prime}>t_{1}$. Then from (14) we deduce that the inequalities $z\left(t_{k}^{\prime}\right)=x\left(t_{k}^{\prime}\right)>0, t_{k}^{\prime}>t_{1}, k \in \mathbb{N}$, hold true. The established inequalities contradict the assumption made.

Theorem 6: Let the following conditions hold:

1. Conditions 1,2 and 3 of Lemma 1 are valid.

2. There exists a constant $L>1$ such that

3. $\quad \liminf _{k \rightarrow \infty} \int_{\tau_{k}}^{\tau_{k}+h} p_{1}(s) d s>1+M+\frac{M\left[q_{2}-q_{1}\right]}{S L+q_{1}-q_{2}}$, where $S=\mathrm{const}>0$,

$$
\int_{0}^{\tau_{k}} q(s) d s \geq \ln L, \quad k \in \mathbb{N}
$$

$$
p_{1}(t)=p(t) \exp \left[\int_{t-h}^{t} q(s) d s\right], t \in \overline{\mathbb{R}}_{+} \cdot
$$

Then all solutions of the problem (2), (3) satisfying the inequalities $\left|x\left(\tau_{k}\right)\right|>S$, $k \in \mathbb{N}$, oscillate.

Proof: Set

$$
x(t)=z(t) e^{-\int_{0}^{t} q(s) d s},
$$

and substitute it into (2) to obtain

$$
\left.\begin{array}{c}
z^{\prime}(t)+p_{1}(t) z(t-h)=b(t), \quad t \neq \tau_{k} \\
\Delta z\left(\tau_{k}\right)=b_{k} z\left(\tau_{k}\right) .
\end{array}\right\}
$$

Taking into account condition 2 of Theorem 6 , we derive the estimate

$$
\left|z\left(\tau_{k}\right)\right|=\left|x\left(\tau_{k}\right)\right| e^{\int^{\tau_{k}} q(s) d s} \geq S e^{\ln L}=S L, \quad k \in \mathbb{N} .
$$


From Lemma 1 it follows that all solutions of the problem (15), (3) for which $\left|z\left(\tau_{k}\right)\right|>S L, k \in \mathbb{N}$, oscillate. The latter immediately yields that all solutions of the problem (2), (3) for which $\left|x\left(\tau_{k}\right)\right| \geq S$, oscillate.

\section{Acknowledgement}

The authors express their gratitude to the referee for the valuable suggestions.

The present investigation was supported by the Bulgarian Ministry of Education,

Science and Technologies under grant MM-511.

\section{References}

[1] Gopalsamy, K. and Zhang, B.G., On delay differential equations with impulses, J. Math. Anal. Appl. 139:1 (1989), 110-122.

[2] Györi, I. and Ladas, G., Oscillation Theory of Delay Differential Equations with Applications, Clarendon Press, Oxford 1991.

[3] Ladde, G.S., Lakshmikantham, V. and Zhang, B.G., Oscillation Theory of Differential Equations with Deviating Arguments, Pure and Applied Mathematics, Vol. 110, Marcel Dekker, New York 1987.

[4] Shevelo, V.N., Oscillations of Solutions of Differential Equations with Deviating Arguments, Naukova Dumka, Kiev 1978. (in Russian) 


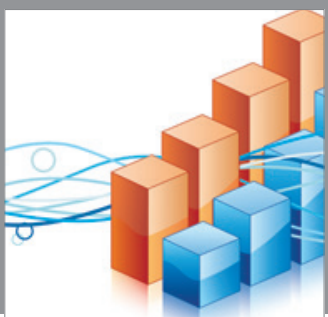

Advances in

Operations Research

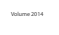

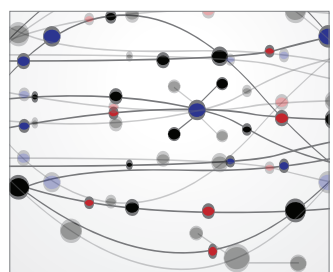

\section{The Scientific} World Journal
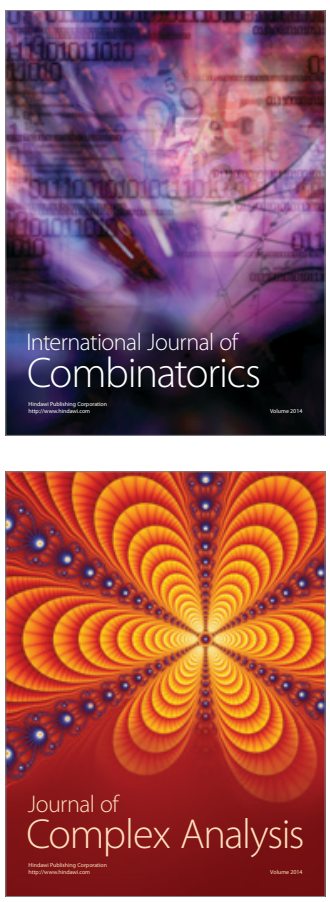

International Journal of

Mathematics and

Mathematical

Sciences
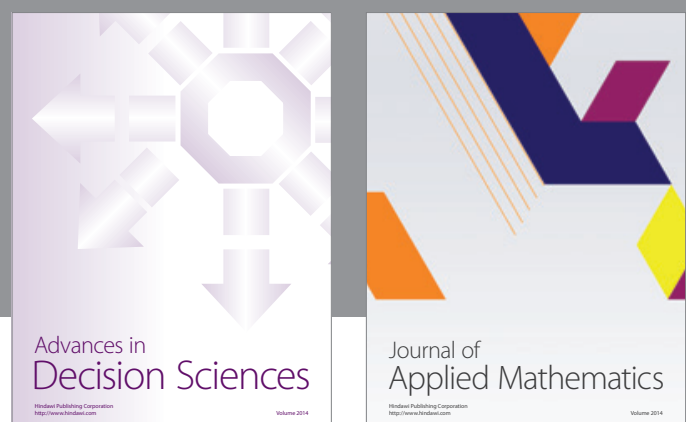

Journal of

Applied Mathematics
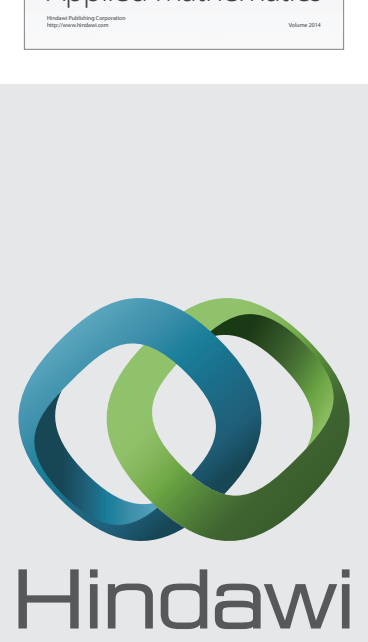

Submit your manuscripts at http://www.hindawi.com
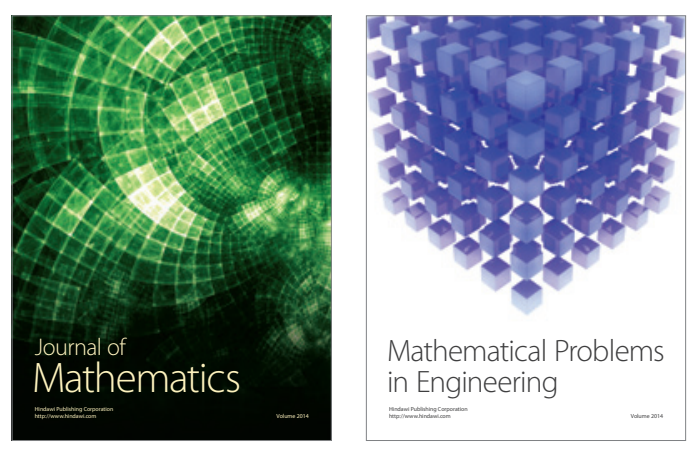

Mathematical Problems in Engineering
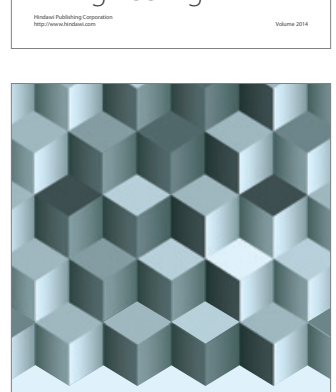

Journal of

Function Spaces
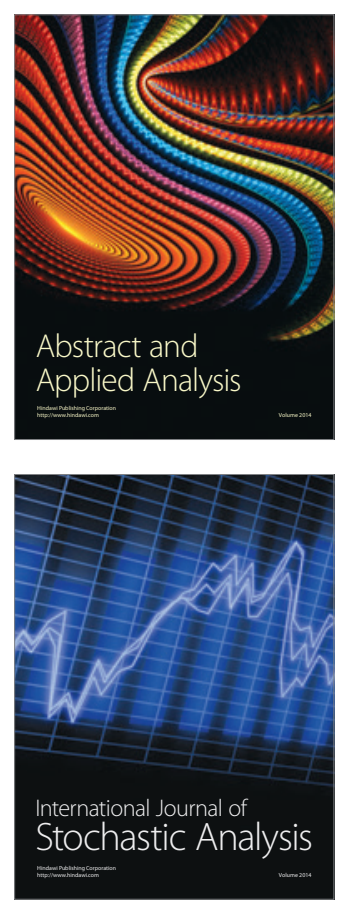

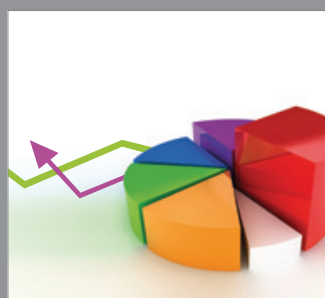

ournal of

Probability and Statistics

Promensencen
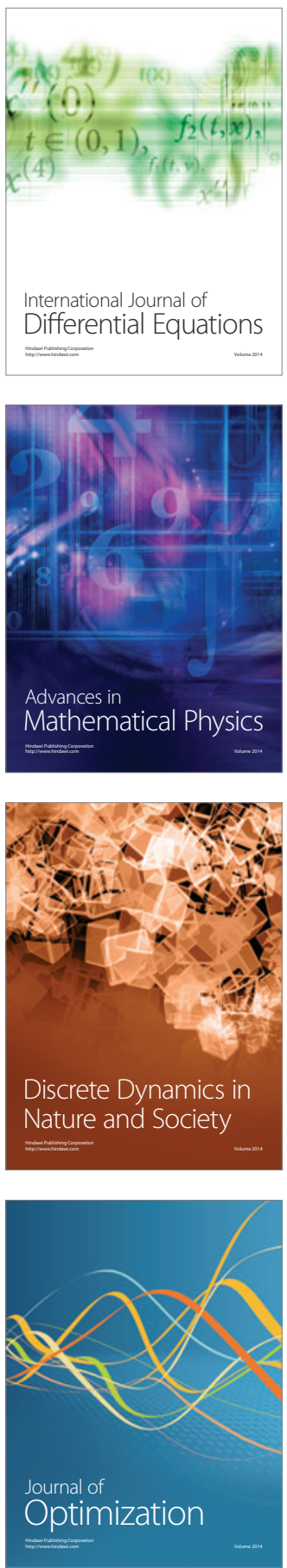